Vortr. schließt damit, daß es also schon jetzt möglich ist, die verschiedenen Nystagmusarten zu gruppieren und sie in ein allgemeines Schema einzuordnen.

32. Heyninx (Brüssel): Die Prüfung des Vestibulum durch Vor- und Rückwärtsgehen bei verschlossenen Augen. Vgl. dieses Archiv Bd. 94, S. 147.

33. Escat (Toulouse) und Vaquier (Tunis): Die Ungleichheit der akustischen Empfindlichkeit für die kontinuierliche Tonreihe. Physiologische Hörkurven. Die Vortr. haben für die versehiedenen Töne von c mit 32 bis $\mathrm{c}^{10}$ mit 32768 Doppelschwingungen (durch Metallsaiten hervorgebracht) den größten Abstand, in welchem jeder Ton noch hörbar ist, ermittelt. Durch Kom. bination der Einzelkurven haben sie eine sehr charakteristisehe Gesamtkurve erhalten. Diese zeigt, daß die Hörweite für die aufeinander folgenden Töne zuenst unmerklich, dann schnell anwächst, um ihr Maximum bei $\mathrm{g}^{6}$ gleich 3072 Doppelschwingungen zu erreichen, worauf sie um vieles schneller wieder abnimmt und an der oberen Hörgrenze, bei $c^{10}$, gleich Null wird. Der ansteigende Teil der Kurve umfaßt 8 , der absteigende 3 oktaven.

Capart Sohn (Brüssel)-Blau.

\title{
IV. Royal Society of Medicine, London. Otologische Abteilung.
}

\section{Sitzung rom 17. Oktober 1913.}

1. Mollison: Ein Fall von Ohrverletzung. Der 43jährige Patient hatte vor 3 Monaten einen so heftigen Schlag von einer Maschine gegen die linke Kopfseite bekommen, daß man zweifelte, ihn am Leben erhalten zu können. Trotzdem genesen, zeigte er folgenden Befund: Lähmung des 3.-7. Hirnnerven auf der linken Seite, Fixation des Augapfels, Erblindung und Hornhautgeschwür. 'Traumatische Atresie des linken Gehörganges, sehr starke Schwerhörigkeit mit Einschränkung an der oberen und unteren Tongrenze und Herabsetzung der rotatorischen Reaktion. Es wurde operativ ein äuBerer Gehörgang horgestellt, worauf das Gehör bedeutend zunahm, die untere Tongrenze normal, die obere fast normal wurde. Trommelfell anscheinend ohne Veränderung, Drehreaktion beiderseits gleich mit deutlich sichtbaren Bewegungen auch des linken Auges, kalorische Erregbarkeit links gesteigert.

In der Diskussion bezeichnen es West und Jenkins als bemerkenswert, dab Patient linkerseits trotz Lähmung des Musc. rectus externus einen Nystagmus nach außen zeigte. Jenkins hat zwei Fälle mit sogenanntem kongenitalem Defekt des Rectus externus in Behandlung; bei beiden kann er auf dem betroffenen Auge wohl einen Nystagmus nach innen, aber keinen solchen nach außen erzeugen. Er schließt hieraus, daß die Läsion in des Vortr. Falle den Nerven nicht an der Schädelbasis getroffen haben konnte, sondern wahrscheinlich eine kortikale war. Dieses ist von Bedeutung, weil Wils on das Nystagmuszentrum in die Hirnminde verlegt hat, während es nach Bárány tiefer liegt.

Stuart-Low spricht die Vermutung aus, dah die nach der Operation noch restierende starke Gehörsherabsetzung auf dem linken ohre des Patienten vielleicht Folge eines organisierten Blutgerinnsels in der Paukenhöhle ist oder möglicherweise, wie die vorhanden gewesene Taubheit überhaupt, eine funktionelle, hysterische Grundlage hat. 
2. Scott: Serös-eitrige Leptomeningitis mit schneller Heilung nach translabyrinthärer Operation. Der 28 Jahre alte, mit chronischer linksseitiger Otitis media pur. behaftete Patient klagte seit letzter Woche über starken, sich steigernden Stirnkopfschmerz, Schwindel bei jeder Kopfbewegung nach links, rasche Zunahme der Schwerhörigkeit und laute Ohrgeräusche. Die funktionelle Untersuchung sprach für eine (letzthin eingetretene) Invasion des Labyrinths bei noch vorhandenen Hörresten. Es wurde daraufhin die Radikaloperation, sowie die Eröffnung des Vestibulum und der Schnecke vorgenommen; breite Fistel des horizontalen Bogenganges, Steigbügel nicht gut zu erkennen, aber Resistenz in der Gegend des ovalen Fensters mit der Sonde fühlbar, Inhalt des Bogenganges dunkelrot, kein Eiter, bei Abtragung der Basis des Modiolus Ausfluß von etwas Cerebrospinalflüssigkeit. Nach anfänglicher Besserung kehrte nach zwei Tagen der Kopfschmerz (jetzt vorzugsweise im Hinterhaupt) zurück, und dazu gesellten sich Retraktion des Kopfes, Nackensteifigkeit und Fieber von $39^{\circ}$. Lumbalpunktat trübe, unter hohem Druck, mit 3500 Polynukleären auf den Kubikzentimeter, aber ohne Mikroben. Am dritten Tage nach der ersten Operation wurde jetzt durch den äußeren Gehörgang ein schmaler Meißel eingeführt und mit zwei leichten Hammerschlägen die gut sichtbare innere Labyrinthwand nach dem Meatus aud. int. zu abgeschlagen. Es entleerte sich sofort eine beträchtliche Menge trüber Liquor, und der Ausfluß dieses dauerte noch gegen 14 Stunden fort. Am Abend des gleichen Tages wurde noch eine zweite Lumbalpunktion gemacht. Von da an verloren sich die Beschwerden und das Fieber schnell; zurzeit bietet die Wundhöhle das gewöhnliche Bild einer in Heilung begriffenen Radikaloperations. höhle.

Diskussion.

Lane hält es für möglich, daß die vom Vortr. in Anwendung gebrachte primäre Hauttransplantation die freie Drainage beeinträchtigt hat.

West macht die Transplantationen nicht mehr, wenn bei der Operation eine Labyrinthfistel gefunden wird, oder wenn auch nur die Kranken an Schwindel gelitten haben. In zwei Fällen glaubte er, den üblen Ausgang geradezu auf die Transplantation beziehen zu können. Das eine Mal hatte er den Lappen über eine dunkle, seiner Meinung nach nicht perforierte Stelle am Bogengang gelegt, und der Patient bekam Labyrinthitis, Meningitis und starb. In dem zweiten Falle trat Exitus ein, trotzdem nur auf den hinteren Teil der Wundhöhle transplantiert worden war.

O'Malley hat sich bei den 8 von ihm gesehenen, verschieden schweren Labyrinthitisfällen in der Behandlung ganz nach den von Neumann und Bárány gegebenen Regeln gerichtet. Er ist kein Freund der Hauttransplantationen, weil er alles, was in einem so gefährlichen Bezirk den freien Sekretabfluß behindert, vermeiden möchte.

Mc. Kenzie berichtet über einen ähnlichen Fall, wie den des Vortr., mit chronischer Eiterung, Labyrinthitis, Labyrinthoperation, zwei Tage später Meningitis, aber mit tödlichem Ausgang. Er fragt sich, $o b$ die Labyrintheroffnung in seinem und auch des Vortr. Falle berechtigt gewesen ist. Seiner jetzigen Anschauung nach würde er sich bei Kranken mit chronischer Mittelohreiterung und labyrinthären Symptomen, die nicht eine totale Aufhebung sowohl der vestibularen als der kochlearen Funktion aufweisen, auf die Radikaloperation beschränken und die Labyrinthoperation gleichzeitig mit ihr nur dann machen, wenn jene beiden Funktionen vernichtet sind, oder sekundär, wenn sich Zeichen von Meningitis einstellen, die, unabhängig vom 
funktionellen Zustand des Labyrinths, die Drainage durch Labyrinth und inneren Gehörgang dringend fordern.

3. Hunter Tod: Trichophyton-Granulom der Ohrmuschel. Der 21jährige Patient hatte vor 7 Jahren einen Herpes tondens der Bauchgegend gehabt und war davon geheilt worden. In der Folge wurde das linke $O h x$ ergriffen, und es bildete sich allmählich eine pilzartige Geschwulst der Muschel und Warzengegend. Diese verschwand bei Röntgenbehandlung in Zeit von 6 Monaten, so daB nur ein Stumpf der Muschel zurückblieb. Die Heilung hat seitdem über ein Jahr lang angehalten. Eine Mittelohreiterung, die mehrmals aufgetreten ist, hatte mit der Trichophyton-Infektion wohl keinen Zusammenhang. Zurzeit bestehen Effloreszenzen noch in der linken Achselhöhle und Brustgegend und werden hier erfolgreich mit Karbolinjektionen und X-Strahlen behandelt. Fortr. bemerkt, daß die Krankheit, die in England nicht vorkomamt, wahrscheinlich von Dänemark aus, wo sie sich häufig vorfinden soll, eingeschleppt worden ist. Eine Schwester des Patienten hatte vor diesem an ihr gelitten. 4. Jenkins und Stebbing: Maligner Tumor des Gehörorgans. Epitheliom (?) mit Umwandlung in Sarkom. 48jähriger Mann, hatte im letzten Sommer eine Drüsenschwellung an der linken Halsseite unter dem Unterkieferwinkel bemerkt, die exstirpiert wurde. Im November AusfluB aus dem Ohre, Zunahme der Schwellung am Hals, herauswuchernder Ohrpolyp, dann Fazialislähmung, Schluckstörungen und Regurgitation genossener Flüssigkeiten durch die Nase, Abmagerung. Untersuchungsbefund: Linke Ohrmuschel durch eine teigige Infiltration vom Schädel abgehoben, walnußgroßer, leicht blutender Polyp aus dem erweiterten Gehörgang herausgewachsen, übelriechender Ausfluß. Unter dem Ohre eine Geschwulst, die nach hinten bis an den hinteren Rand des Kopfnickers, nach vorn fast bis zur Mittellinie des Larynx reichte und hier mit einem scharfen Rande abschnitt. Geschwollene Drüsen über der Clavicula. Parese (frische) des Jinken Abduzens, Zunge nach links vorgestreckt, Lähmung der linken Hälfte des weichen Gaumens, sowie der Stimmbandadduktoren. Parese und Muskelschwund des linken Trapezius. Bedeutende Schwerhörigkeit linkerseits vom Mittelohrtypus. Der Tod trat am 1. April nach voraufgegangenen mehrwöchigen starken Schmerzen ein. Bei der Sektion zeigten sich der Nerv. hypoglossus, der N. laryngeus externus und der spinale Teil des $\mathrm{N}$. accessorius in die Halsgeschwulst aufgegangen, während der Recurrens, Vagus und-Sympathicus frei geblieben waren. Äußerer Gehörgang und Mittelohr von Geschwulstmassen erfüllt, mit Zerstörung des Paukenhöhlenbodens, beginnendem Durchbruch in die mittlere Schädelgrube und Ausbreitung, wie es schien, auch nach vorn längs der Tuba. Der Nerv, facialis ging durch die Geschwulst. Ihrem histologischen Charakter nach ergab sich diese als ein Sarkom.

In der Diskussion bemerkte Marriage, daß die von ihm im September entfernten Halsdrüsen und ebenso ein im Dezember durch Probeexzision entnommenes Geschwulststück karzinomatöser Natur gewesen waren.

Gray sucht, diese beiden Befunde in Ưbereinstimmung zu bringen. Auf Mäuse überimpftes Karzinom kann gelegentlich eine sarkomatöse Reaktion in den Geweben hervorrufen. Er selbst hat von einem Lippenepitheliom eine epitheliomatöse Metastase am Bein gesehen, die später sarkomatös geworden ist. Das Bindegewebe reagiert manchmal in so intensiver Weise, daß die Epithelzellen verdrängt werden und sich an ihrer Stelle eine maligne Bindegewebswucherung entwickelt. Das umgekehrte Verhalten, Übergang von Sarkom in Karzinom, ist nicht beobachtet worden.

Jenkins sagt im Schlußwort, daß die demonstrierten Präparate 
keinesfalls von einem Epitheliom herstammen können. Es handelt sich um ein Sarkom oder Endotheliom, am wahrscheinlichsten um das erstere.

\section{Sitzung vom 21. November 1913.}

1. Kelson: Eine neue Tubenbougie. Die Bougie ist an einer gleitenden Skala befestigt, und es ist die Einrichtung getroffen, daß die Distanz, um welche sie über das Ende des Katheters hinausragt, sich selbsttätig auf der Skala anzeigt. Da das ganze Instrument einschließlich der Bougie aus Metall besteht, kann es leicht durch Kochen sterilisiert werden; man kann aber auch Bougies aus Kautschuk oder einem anderen Stoff verwenden.

In der D iskussion wird von verschiedener Seite die Befürchtung ausgesprochen, mit so dünnen Stahlbougies, wie die gezeigten, die Tubenwand $z u$ verletzen und falsche Wege zu verursachen. Die Indikation für die Bougierung wird eng begrenzt. Powell meint, daß nur in einer kleinen Zahl von Fällen der Katheterismus allein zur Öffnung der Tuba nicht ausreichen dürfte. Scott verwendet die Bougierung manchmal, um dicken Schleim aus dem Wege zu räumen, der das Durchstreichen der Luft verhindert. Mole hat von ihr bei Kranken Nutzen gesehen, bei denen die durch die Luftdusche herbeigeführte Hörverbesserung immer nur vorübergehend war. Demgegenüber betont $K$ elson in seinem Schlußwort die Notwendigkeit der Bougierung, wenn die Luftdusche durch den richtig sitzenden Katheter wegen irgendeines Hindernisses nicht gelingt. Fimphysem entsteht nicht durch Terletzung der Tubenwand, sondern durch solche des Gewebes um das Tubenostium, wenn das einzuführende Instrument, sei es die Bougie oder ebenso der Metallkatheter, sich in falscher Lage befindet.

2. Muecke: Lateralsinus-Thrombose mit Pyämie. Der 46 Jahre alte Patient war vor 9 Wochen an akuter Otitis media mit schnell eintretender Spontanperforation erkrankt. Vor 1 1/2 Wochen Stocken des Ausflusses, zugleich Kopfschmerzen, Uंbelbefinden, Brechreiz. Bei der Aufnahme waren die einzigen Klagen über Schmerzen in der linken Halsseite, wo sich auch objektiv eine diffuse Rötung und empfindliche Schwellung längs des Verlaufes der Vena jugularis int. vorfand. T. $39^{\circ}$, P. 100. Kein Schüttelfrost in der Anamnese. Warzengegend normal, hintere obere Gehörgangswand gerötet und geschwollen, profuse Eiterung aus einer kleinen Öffnung im oberen Trommelfellabschnitt. Im Blut stark vermehrte polynukleäre neutrophile und große hyaline Zellen, Reinkultur von Streptokokken aus dem Fingerblut. Cerebrospinalflüssigkeit normal. Operation: Simus gangränös, wurde nach beiden Seiten hin ausgeräumt. Thrombose der Vena jugularis int., Ligatur dieser, sowie der ebenfalls thrombosierten Vena facialis, Spaltung des oberen Jugularisendes und Einnähen in den oberen Wundwinkel, Durchspullungen vom Sinus aus. Der Verlauf war in der ersten Zeit gut, bis auf eine leichte Fazialislähmung am 2. Tage, die am 4. Tage komplett wurde, und eine einmalige Temperatursteigerung auf $39^{\circ}$. Bei dieser Gelegenheit und später noch fünfmal bei hohen Temperaturanstiegen wurde eine Vakzine injiziert. Das Blut war 10 Tage nach der Operation baktexienfrei, 12 Tage später Thrombose der linken, dann nach 4 Tagen desgleichen der rechten Vena poplitea. Nach weiteren 10 Tagen Sehmerzen in der rechten Nierengegend, am nächsten Tage Blut und Eiter im Urin, bald darauf Oystitis und linksseitige Epididimo-Orchitis. Zuletzt stellte sich noch eine akute Peritonitis ein, zu deren Hebung die Laparatomie gemacht wurde. Von da an begann der Kranke sich langsam $\mathrm{zu}$ bessern und wurde 14 Wochen nach der ersten Operation zur Erholung an die Meeresküste geschickt. 
D isk us sion.

Lane sieht die starke Halsschwellung, die in dem die Jugularis miteinbegreifenden Absze $\beta$ ihre Ursache hatte, als einen ungewöhnlichen Zug des Falles an.

Scott hat nie einen so schweren Fall von echter Pyämie genesen sehen. Die Komplikation sogar noch mit Peritonitis macht ihn fast zu einem Unikum.

Jenkins fragt, ob Vortr. den Fall nicht eher als solchen von Septizämie auffaß $\mathrm{t}$, da doch der gesamte Blutstrom mit den Infektionskeimen beladen war. Er macht auf die individuell sehr verschiedene Resistenz der Streptokokkeninfektion gegenüber aufmerksam.

Kels on erkundigt sich, ob die verwendete Vakzine eine autogene war, und $o b$ Vortr. ihr einen wesentlichen EinfluB auf die Heilung zuschreibt.

Powell hebt die Tatsache hervor, wie lange derartige Fälle ohne beunruhigende Symptome verlaufen können. Er erwähnt eine Kranke, die wochenlang wegen, ,Rheumatismus" behandelt worden war, und bei der dann, als sich Cerebralsymptome und ein Abszeß am Knie einstellten, die Operation eine auf die andere Seite übergreifende septische Thrombose des Sinus transversus und später die Sektion auBerdem eine Thrombose des Sinus rectus, petrosus und cavernosus und eine Nekrose des Felsenbeins aufdeckte.

Muecke (Schlußwort) gibt an, daß unter 80 Fällen des London Hospital ein HalsabszeB, wie der beschriebene, nur viermal vorgekommen ist, und zwar ausschließlich in verschleppten Fällen. Den Befunden nach hat es sich bei seinem Kranken sowohl um eine Septizämie als Pyämie gehandelt. Die Vakzine war eine frisch bereitete autogene, ihre Injektion hatte jedesmal einen raschen Temperaturabfall zur Folge. Diagnostische Irrtümer, daß nämlich die Erkrankung für ein Typhoid oder eine Lungenaffektion gehalten wird, ereignen sich während des Lebens häufig und lassen sich oft in den Seltionsprotokollen nachweisen. Die Fazialislähmung nach der Operation, trotzdem der Nerv bei ihr nicht berührt worden war, konnte vielleicht auf die nachträgliche Tamponade bezogen werden.

3. Muecke: Lateralsinus-Thrombose und Abduzenslähmung. Patientin 15 Jahre alt. Seit 5 Tagen starker Kopfschmerz, Krankheitsgefühl, Übelkeiten. Zwei Schüttelfröste. Seit dem Tage vorher Lähmung des gleichseitigen Musc. rectus oculi externus. Warzengegend normal. Die Operation, die, wie im vorigen Falle, ausgeführt wurde, ergab eine Gangrän des Sinus an der Umbiegungsstelle und eine bis zur Einmündung der Vena facialis communis hinabreichende Thrombose der Jugularis. Glatter Heilungsverlauf bis auf eine akute Synovitis am Ellbogengelenk. Zur Zeit der Entlassung war die Augenmuskellähmung ein wenig zurückgegangen.

In der $\mathrm{D}$ iskussion wurde die Frage der Abduzenslähmung bei Ohraffektionen eingehend erörtert. I a ne erwähnt ihr Forkommen bei Kindern ohne sonstige Komplikation als eine Mastoiditis, sowie andererseits neben I abyrinthitis und Hirnabszeß. Jenkins bemerkt, daß in den nicht zahlreichen Fällen, die er gesehen hat, die Lähmung fast immer von akuter Mastoiditis begleitet war. Der Ausgang nach gemachter Mastoidoperation war immer in Heilung. J. erklärt die Lähmung in der Weise, dab auf Grund der Reizung vom Warzenfortsatz aus ein vermehrter Blutzufluß zur AuBenseite der Dura an der hinteren Pyramidenfläche und daher eine Gewebsverdickung derselben eintritt, die nur gering zu sein braucht, aber doch den Nerven durch Kompression schädigen kann. Scott hatte die ersten Fälle von Abduzenslähmung neben Labyrinthitis beobachtet und sie auf Übergreifen der Infektion vom Labyrinth auf die Meningen und auf den Abduzens selbst zurückgeführt. Diese Auffassung ist aber durch die Beobachtung 
mehrerer Fälle von seröser Meningitis als unrichtig erwiesen worden, da bei ihnen zwar der Nerv gelähmt, das I abyrinth aber nicht beteiligt war und bei der einige Male gemachten Sektion keine entzündliche Veränderung am Nerven gefunden wurde. Er sieht die Ursache der Lähmung jetzt in dem gesteigerten intrakraniellen Druck und der durch ihn bedingten Kompression des Nerven bei seinem Durchtritt durch die Dura mater, ohne daß diese selbst oder der Nerv von Entzündung ergriffen sind. Hierbei handelt es sich um transitorische Formen der Abduzenslähmung, die sowohl auf der Seite des erkrankten Ohres als auf der entgegengesetzten Seite sich zeigen können. U $r$ ban Pritchard ist am ehesten geneigt, als ätiologischen Faktor eine zirkumskripte Meningitis anzunehmen. Fr hat nicht gefunden, daß diese Fälle gerade besonders mit starkem Kopfschmerz einhergingen, der auf eine intrakranielle Drucksteigerung hinweisen würde. Nach der Mastoidoperation bildet sich die Lähmung regelmäßig zurück, indem die Blutentziehung und der Gegenreiz heilend auf die umschriebene Kongestion oder Entzündung der Meningen einwirken. Muecke (Schlußwort) bemerkt, daß in seinem Falle kein äuBerliches Zeichen von Fntzündung des Antrum vorhanden war, und daB selbst stärkster Druck in der Warzengegend keinen Schmerz auslöste. Er hat durch seine Mitteilung andere davon abhalten wollen, etwa unnötigerweise die Dura zu inzidieren.

Blau.

\section{Atti della Clinica oto-rino-laringoiatrica della Reale Uni- versità di Roma.}

Diretta dal Prof. Gh. Ferreri. Anno X. 1912.

Der 10. Jahrgang der bekannten italienischen Tahresberichte ist nun erschienen und reiht sich an Reichtum des Inhalts und an Gediegenheit der Leistungen würdig seinen Vorgängern an. Die Jahrbücher haben sich schon lange das Bürgerrecht in der internationalen Literatur unseres Faches erworben, und jeder Jahrgang liefert ein neues Blatt in den Lorbeerkranz der italienischen wissenschaftlichen Fachliteratur. Theoretische Studien, klinische Beobachtungen, anatomisch-pathologische Untersuchungen, historische Exkursionen und experimentelle Forschungen werden in abwechslungsreicher Reihenfolge von einer auserlesenen Schar wissenschaftlicher Mitarbeiter geboten. Der I,eiter der Jahrbücher, Prof. Ferreri, kann mit Genugtuung und edlem Selbstbewußtsein auf diese seine 10 jährige Tätigkeit zurückblicken, durch welche er seinem Vaterlande unsterbliche Dienste geleistet, die Fachkollegen aller Nationen zu Dank verpflichtet hat. Diesem unermüdlichen und ausdauernden Arbeiten der italienischen Kollegen, Ferreri voran, ist es auch zu verdanken, dab unser Spezialfach in Italien sich jene Stellung unter den medizinischen Wissenschaften und eine solche Würdigung und Anerkennung bei den Behörden errungen hat, wie es ihm seiner wissenschaftlichen und praktischen Leistungen wegen gebübrt.

In der Vorrede zum letzten Jahrgang hebt auch Ferreri die grobartigen Fortschritte unserer Disziplin hervor, seitdem es den Bemühungen De Ross is gelungen ist, derselben eine ihrer würdige Stätte in der großartigen Poliklinik Humbert I. in Rom zu erwirken. Erzogen in der Schule dieses Meisters und durchdrungen von dessen Begeisterung hat als sein wuirdiger Nachfolger Gh. Ferreri es sich zur Aufgabe gemacht, nicht nur das Erworbene zu erhalten, sondern auch das Ansehen der Spezialität noch möglichst zu heben. Unterstützt durch die Munifizenz des Unterrichtsministers und der medizinischen Fakultät ist es F. gelungen, das Institut auf jene Stufe der Vollkommenheit zu bringen, auf der es jetzt seine musterhafte Tätigkeit entfaltet. Referent hat Gelegenheit gehabt, sich durch persönliche 